\title{
Effect on Growth Velocity of Dose-Frequency of Growth Hormone (GH) Treatment in Idiopathic GH Deficient Prepubertal Children: The Kabi Pharmacia International Growth Study (KIGS) Experience
}

Pierre G Chatelain, Oliver Guilbaud, Patric Wilton and Rolf Gunnarsson on behalf of the KIGS Board Department of Pediatrics, Hôspital Debrousse (PC), Lyon, France, Kabi Pharmacia, (OG, PW, RG), Stockholm, Sweden

Growth velocity (GV) in response to recombinant growth hormone (GH) treatment (Rx) was analysed using Kabi Pharmacia International Growth Study (KIGS) data base. Idiopathic GH deficiency (IGHD) was based on classical criteria: short stature, slow growth velocity; GH peak response at 2 provocative tests below $10 \mathrm{ng} / \mathrm{mL}$; thyroid and adrenal status were based on conventional hormonal evaluation (local assays). Organic GHD was ruled out by brain imaging. 1,256 prepubertal IGHD GH treated patients in the KIGS data base were analysed. At start of $\mathrm{Rx}$ mean chronological age (CA) was 7.3 years and mean height standard deviation score (SDS) was -2.7. Mean number of injections was 6/week and mean GV were 8.2 and $6.9 \mathrm{~cm} / \mathrm{y}$ during $\mathrm{Rx}$ y 1 and 2, respectively. Out of ten potential predictors, multivariance analysis resulted in a five predictors model of growth response to Rx: target height (SDS), height SDS for CA, CA at start, GH dose and GH injection frequency (R-square $=0.3707$ ). The effect of GH dose at 3 versus 7 injections/week according to CA are shown in the figure. The two predictors not shown are fixed at their mean value. Conclusions: These data provide useful information for optimising GH treatment in IGHD and predicting growth response.
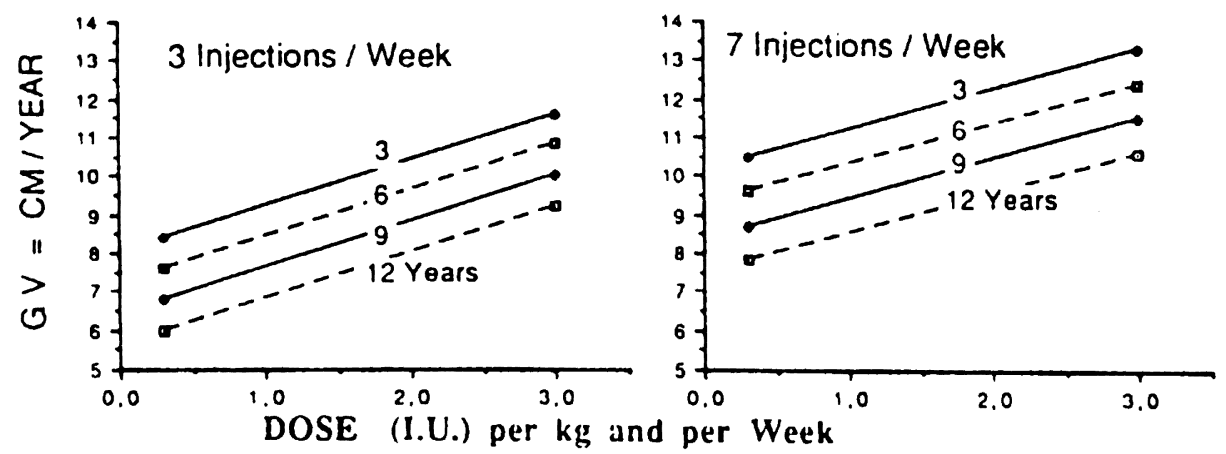

Fig. 